\title{
Excitation temperature, degree of ionization of added iron species, and electron density in an exploding thin film plasma
}

\author{
S. Y. SUH* and R. D. SACKS \\ Department of Chemistry, University of Michigan, Ann Arbor, Ml 48109, U.S.A.
}

(Received 7 April 1981)

\begin{abstract}
Time and spacially resolved spectra of a cylindrically symmetric exploding thin film plasma were obtained with a rotating mirror camera and astigmatic imaging. These spectra were deconvoluted to obtain relative spectral emissivity profiles for nine $\mathrm{Fe}(\mathrm{II})$ and two $\mathrm{Fe}$ (I) lines. The effective (electronic) excitation temperature at various positions in the plasma and at various times during the first current halfcycle was computed from the Fe(II) emissivity values using the Boltzmann graphical method. The $\mathrm{Fe}(\mathrm{II}) / \mathrm{Fe}(\mathrm{I})$ emissivity ratios together with the temperature were used to determine the degree of ionization of Fe. Finally, the electron density was estimated from the Saha equilibrium. Electronic excitation temperatures range from $10,000-15,000 \mathrm{~K}$ near the electrode surface at peak discharge current to $7000-10,000 \mathrm{~K}$ at 6-10 $\mathrm{mm}$ above the electrode surface at the first current zero. Corresponding electron densities range from $10^{17}-10^{18} \mathrm{~cm}^{-3}$ at peak current to $10^{13}-10^{16} \mathrm{~cm}^{-3}$ near zero current. Error propagation and criteria for thermodynamic equilibrium are discussed.
\end{abstract}

\section{INTRODUCTION}

THE HIGH-TEMPERATURE plasmas produced by the rapid electrical vaporization of $\mathrm{Ag}$ thin films recently have been used as atomization cells and excitation sources for the direct determination of selected elements in micro-size powder samples [1]. Complete vaporization of small-particle powder samples as refractory as $\mathrm{ZrC}$ (boiling point = $5370 \mathrm{~K}$ ) was observed. This, coupled with minimal concomitant effects and detection limits in the $10^{-9}$ to $10^{-12} \mathrm{~g}$ range, suggests that exploding thin film techniques could, with further development, provide a viable solution to the multielement determination problem with solid, non-conducting samples. Recently reported studies [2] using high-current wave shaping techniques for the temporal decoupling of the atomization and excitation processes suggest that order-of-magnitude improvements in limits of detection can be obtained with minimal experimental difficulty. Other recent work [3] has shown that with the careful choice of discharge conditions and time-gated radiation measurements, samples with particles ranging in size from less than 5 to about $50 \mu \mathrm{m}$ in diameter can be analyzed with no apparent particle size effects. Thus, sample preparation is reduced to a 5-10 min grinding operation in a laboratory mill.

Despite these attractive features of exploding thin film atomization and excitation and a genuine need for improved direct analytical methods for solid samples, a dearth of fundamental information regarding the properties of these high-temperature, atmospheric pressure discharges has frustrated experiment design and the development of routine analytical procedures. There are no published reports concerning temperature, degree of ionization, electron number density and related properties of these plasmas. While there are several studies [4-7] in which temperature is measured

[1] E. M. ClaRK and R. D. SACKS, Spectrochim. Acta 35B, 471 (1980).

[2] S. Y. SuH, R. J. Collins and R. D. SACKS, Appl. Spectrosc. 35, 42 (1981).

[3] J. Goldberg, J. SWAN and R. SACKs, VII Annual Meeting Federation of Analytical Chemistry and Spectroscopy Societies. Philadelphia (1980).

[4] H. JÄGER and W. LoChte-Holtareven, Exploding Wires (Edited by W. G. Chace and H. K. MoORE), Vol. 4, p. 41. Plenum, New York (1968).

[5] B. YA'Akobi, A. S. Kaufman and P. Avivi, Proc. 7th Int. Conf. on Ionization Phenomena in Gases, Vol. 1, p. 799. Graderinska Knjiga, Belgrade $\cdot(1965)$.

[6] H. Bartels and J. BortfeldT, Exploding Wires (Edited by W. G. Chace and H. K. MoORE), Vol. 3, p. 9. Plenum, New York (1964).

[7] E. B. Mayfield, Exploding Wires (Edited by W. G. Chace and H. K. Moore), Vol. 1, p. 147. Plenum, Ne:r York (1959).

\footnotetext{
*Present address: Bell Laboratories, 555 Union Blvd., Allentown, PA 18103, U.S.A.
} 
for exploding wire plasmas, there is little agreement among the reported values, and usually few experimental details are provided. In addition, in most of these studies, time and/or spacial resolution were not employed. Since these plasmas show extreme temporal and spacial heterogeneity, such measurements of plasma properties are indeed naive.

While the exploding thin-film plasmas may not be in local thermodynamic equilibrium (LTE), so little is known about them that any indication of plasma temperature. even with very restricted definition, would be most useful in designing further experiments. Empirical degree-of-ionization values for analyte species have direct analytical utility in establishing analysis line selection criteria as well as providing a means for an order-of-magnitude estimate of electron number densities.

The experiments reported here used atomic emission intensities and the Boltzmann graphical method to estimate an effective electronic excitation temperature over a limited range of excitation energies. A direct comparison of neutral atom and ion line intensities from a thermometric species together with the tacit and perhaps naive assumption of LTE is used to estimate degree of ionization. Finally, electron number densities are estimated from the degree of ionization and the plasma temperature. It should be noted that these studies were not designed to provide detailed temperature mappings of the plasma but rather to develop measurement techniques and preliminary data for these interesting and potentially very useful radiation sources.

\section{Measurement Theory and Error Propagation}

The reliable determination of electronic excitation temperature is crucial since degree of ionization and electron number density are derived in part from the electronic excitation temperature provided that LTE can be assumed and multiple charged ions are relatively infrequent. The Boltzmann graphical method of temperature determination [8] was chosen for two reasons. First, greater precision is obtained than with the two-line method. This is particularly important for exploding thin film plasmas since it was necessary to use the much more intense ion lines rather than neutral-atom lines. Transition probability data for the former are limited both in availability and in reliability. Second, under favorable circumstances, the Boltzmann graphical method can provide some insight into the degree of equilibrium associated with the plasma.

\subsection{Temperature}

For a radiation source with a symmetry axis, the electronic excitation temperature is defined in terms of the time and spacially dependent spectral emissivity $J(\bar{r}, t, \lambda)$ and the electronic population distribution:

$$
J(\bar{r}, t, \lambda)=\frac{n(\bar{r}, t) h c g A \exp (-E / k T)}{4 \pi \lambda U(T)}
$$

Here, $A, \lambda, g$ and $E$ are the transition probability, spectral wavelength, statistical weight and excitation potential, respectively, for the transition of interest, and $h, k, T$ and $U(T)$ are Planck's constant, Boltzmann's constant, temperature and partition function, respectively. The term $n(\bar{r}, t)$ is the total species number density for a given time $t$ and distance $\bar{r}$ from the discharge axis. Casting this in logarithmic form and replacing $J, A$ and $n$ with relative values $J^{\prime}, A^{\prime}$ and $n^{\prime}$, equation (2) is obtained:

$$
\ln \left[\frac{\lambda J^{\prime}(\bar{r}, t, \lambda)}{g A^{\prime}}\right]=\ln \left[\frac{n^{\prime}(\bar{r}, t)}{U(T)}\right]-\frac{E}{k T}
$$

Since $n^{\prime}(\bar{r}, t)$ and $U(T)$ are transition independent, $\ln \left(\lambda J^{\prime} / g A^{\prime}\right)$ is a linear function of

[8] F. Cabannes and J. Chapelle, Reactions Under Plasma Conditions (Edited by M. Venugopalan), Vol. I, p. 397. Wiley-Interscience, New York (1971). 
excitation energy $E$. Thus, if the left side of equation (2) is plotted vs excitation energy for a series of spectral lines of a particular thermometric species, a straight line is obtained provided that the plasma is in LTE, and both stimulated emission and self-absorption are negligible. The temperature $T$ is determined from the slope of the line.

Reliable temperature determination by the Boltzmann graphical method requires transitions with widely differing upper level excitation energy. It should be noted that an estimate of the slope based on experimental points at the curve extremities is made with greater precision than an estimate based on points scattered along the line.

\subsection{Degree of ionization}

If equation (1) for a singly ionized thermometric species is solved for the particle number density and the result divided by a similar expression for the neutral-atom density, equation (3) is obtained:

$$
\frac{n_{+}^{\prime}}{n_{0}^{\prime}}=\frac{\left(J^{\prime} \lambda U\right)_{+}\left(g A^{\prime}\right)_{0}}{\left(J^{\prime} \lambda U\right)_{0}\left(g A^{\prime}\right)_{+}} \exp \left(\frac{E_{+}-E_{0}}{k T}\right)
$$

where the subscripts + and 0 refer to the ion and neutral-atom values, respectively. If the relative transition probabilities for the neutral atom and ion spectra of an element are available on the same scale, the number density ratio can be estimated from the measured ratio of relative spectral emissivity values.

The degree of ionization $\alpha$ can be obtained from

$$
\alpha=\frac{n_{+}^{\prime}}{n_{+}^{\prime}+n_{0}^{\prime}}=\frac{\gamma}{1+\gamma}
$$

where $\gamma=n_{+}^{\prime} / n_{0}^{\prime}$. Multiple charged ions are assumed to be absent.

\subsection{Electron number density}

Once the number density ratio $n_{+}^{\prime} / n_{0}^{\prime}$ and the plasma temperature are determined, the electron number density $n_{e}$ is found from the Saha equation [9];

$$
n_{e}=\frac{2 n_{0}^{\prime} U_{+}}{n_{+}^{\prime} U_{0}} \frac{(2 \pi m k T)^{3 / 2}}{h^{3}} \exp \left(-\frac{X}{k T}\right)
$$

where $m$ and $X$ are the electron mass and the ionization potential of the thermometric species, respectively.

\subsection{Error propagation}

For a two-point Boltzmann graph (two-line method [10]), equation (2) can be written as

$$
\ln \tilde{J}=\ln (\tilde{g} \tilde{A})-\ln \tilde{\lambda}-\frac{\Delta E}{k T},
$$

where $\tilde{J}, \bar{g} \tilde{A}$ and $\tilde{\lambda}$ are ratios of the relative values from the two transitions, and $\Delta E$ is the difference in upper level excitation energy. From equation (6), an expression for the relative temperature uncertainty $\epsilon_{T}$ is obtained by differentiation:

$$
\epsilon_{T}=\frac{k T}{\Delta E}\left(\epsilon_{A}^{2}+\epsilon_{J}^{2}\right)^{1 / 2}
$$

Here, $\epsilon_{A}$ and $\epsilon_{j}$ are the relative uncertainties associated with the relative transition

[9] H. R. GRIEM, Plasma Spectroscopy. McGraw-Hill, New York (1964).

[10] W. Lochte-Holtgreven, Rept. Progr. Phys. 21, 312 (1958). 
probability ratio and the relative emissivity ratio, respectively. While $\epsilon_{T}$ increases linearly with plasma temperature, higher temperature may result in more intense lines from higher levels of excitation thus permitting the use of a line pair with greater $\Delta E$.

For $M$ replicate measurements of relative emissivity ratio, equation (7) is modified to give

$$
\epsilon_{T}=\frac{k T}{\Delta E}\left(\epsilon_{A}^{2}+\frac{\epsilon_{J}^{2}}{M}\right)^{1 / 2}
$$

Thus, the ultimate precision $\epsilon_{T}^{0}$ for the two-line method is limited by the uncertainty in the transition probability ratio.

$$
\epsilon_{T}^{0}=\lim _{M \rightarrow x} \epsilon_{T}=\frac{k T}{\Delta E} \epsilon_{A}
$$

For the Boltzmann graphical method using $N$ spectral lines, $N(N-1) / 2$ line pairs can be formed, but with only $N-1$ degrees of freedom. Then, the relative temperature uncertainty $\epsilon$ is given by

$$
\epsilon_{T}^{*}=\frac{\epsilon_{T}}{(N-1)^{1 / 2}}
$$

The ultimate precision for the Boltzmann graphical technique is not limited by the random uncertainties $\epsilon_{A}$ of the individual line pairs, as with the two-line method:

$$
\lim _{N \rightarrow \infty} \epsilon_{T}^{*}=0
$$

This is an important advantage of the method. Evidently an overall systematic error that affects all the transition probabilities in the same way cannot be removed by this approach.

An error propagation expression $\epsilon_{n}$ for the ratio $\gamma$ is obtained by differentiation of the logarithmic form of equation (3):

$$
\epsilon_{n}=\left[\epsilon_{J}^{2}+\epsilon_{A}^{2}+\left(\frac{\Delta E}{k T} \epsilon_{T}\right)^{2}\right]^{1 / 2}
$$

This is combined with equation (7) to give

$$
\epsilon_{n}=\left(2 \epsilon_{J}^{2}+2 \epsilon_{A}^{2}\right)^{1 / 2}
$$

Thus, the uncertainties in the emissivity and transition probability ratios propagate with amplification. If $L$ independent neutral atom-ion line pairs are used, $\epsilon_{n}$ is reduced by $L^{-1 / 2}$.

The relative uncertainty in $\alpha$ can be found by differentiation of equation (4):

$$
\epsilon_{\alpha}=\frac{\epsilon_{n}}{(1+\gamma)^{2}}
$$

Thus, $\epsilon_{\alpha}$ decreases with increasing ionization.

For the electron number density the logarithmic form of equation (5) is differentiated to give

$$
\epsilon_{n_{e}}=\left\{\epsilon_{n}^{2}+\left[\frac{9}{4}+\left(\frac{X}{k T}\right)^{2}\right] \epsilon_{T}^{2}\right\}^{1 / 2} .
$$

Note that the temperature precision is more critical than that of the number density ratio, and that lower ionization potential results in smaller $\epsilon_{n_{e}}$. 


\section{Apparatus ANd TeChniques}

Several experimental techniques and hardware components were developed to facilitate these measurements. First, circular thin films and a coaxial electrode configuration were designed to produce plasmas with a symmetry axis. This permits straightforward deconvolution of measured intensity profiles with the Abel integral equation. Second, since exploding thin film plasmas are very heterogeneous in both time and space, an optical system was designed using a streaking camera with astigmatic imaging to obtain photographic spectra with high temporal and spacial resolution. Third, an optical density-to-intensity conversion procedure was developed which yields spacially resolved intensity data integrated over the spectral line profile. Finally, a high efficiency data massage and numerical integration routine was developed to convert intensity profiles into spectral emissivity values.

\subsection{Apparatus}

The high-voltage, capacitive discharge circuit and the associated triggering electronics have been described in detail[2]. The discharge chamber also is similar to previous designs [11] except more precise vertical adjustment $( \pm 0.25 \mathrm{~mm})$ was provided.

The coaxial electrode configuration and substrate support are shown in Fig. 1. The thin $\mathrm{Ag}$ film on a polycarbonate membrane filter substrate [1] is supported on a $5.7 \mathrm{~cm}$ dia. nylon cylinder and clamped under a $5.1 \mathrm{~cm}$ o.d., $1.9 \mathrm{~cm}$ i.d., $3.0 \mathrm{~mm}$ thick graphite ring outer electrode. This electrode is press fit in a cylindrical copper holder. A thin plastic ring (not shown in Fig. 1) is cemented to the top surface of the ring electrode. This prevents sparkover to the top surface and confines the discharge current path to the inner surface of the ring. The $2.4 \mathrm{~mm}$ dia. graphite center electrode is mounted flush with the surface of the nylon support. There is no direct electrical connection between the $\mathrm{Ag}$ thin film and the center electrode. However, when the capacitive discharge is initiated, dielectric breakdown occurs through the pores of the $0.01 \mathrm{~mm}$ thick membrane filter. This technique was experimentally straightforward and very reliable. The time required for this initial breakdown is less than the $0.5 \mu$ s resolution of the oscilloscope used to monitor the discharge current. The electrodes are connected to the discharge circuit by a $1.5 \mathrm{~m}$ long coaxial cable constructed from tinned $\mathrm{Cu}$ braiding.

\subsection{Time-gating, spacially resolving optical system}

Since the plasma expands rapidly normal to the substrate surface, meaningful measurements of relative emissivity require that horizontal ( $x$ axis) intensity profiles

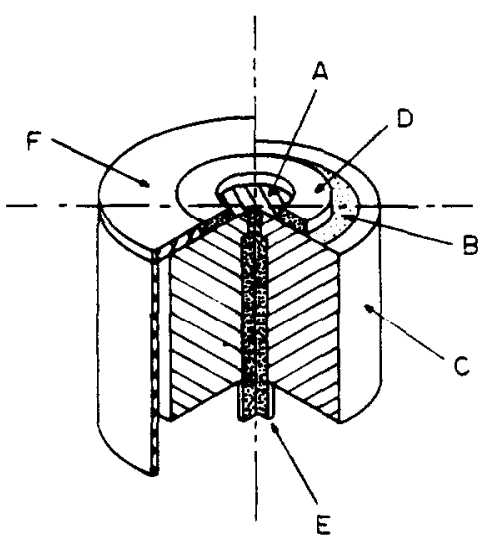

Fig. 1. Coaxial electrode configuration, A, thin Ag film; B, polycarbonate membrane filter substrate; $C$, nylon cylinder body; D, graphite ring outer electrode; $E$, graphite center electrode;

F, copper ring electrode holder.

[11] D. V. DuChane and R. D. SACKs, Anal. Chem. 50, 1752 (1978). 


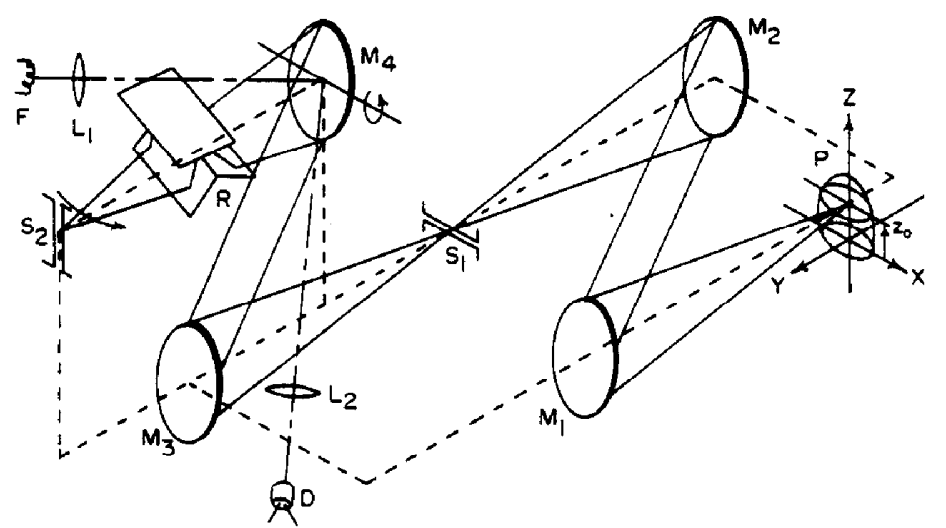

Fig. 2. Optical system using astigmatic imaging for temporal and spacial resolution. Side-by-side spherical mirrors $M_{1}$ and $M_{2}$ image a circular slice of the plasma $P$ onto horizontal slit $S_{1}$. Over-and-under spherical mirrors $M_{3}$ and $M_{6}$ image $S_{1}$ onto the plane of spectrograph slit $S_{2}$ after a $90^{\circ}$ rotation by image rotator $R$. The discharge is triggered when an image of projection lamp filament $F$ sweeps across photodiode $\mathrm{D}$ via $M_{4}$ and lenses $\mathrm{L}_{1}$ and $\mathrm{L}_{2}$.

across the plasma be obtained with vertical ( $z$ axis) spacial resolution as well as temporal resolution. Since the measured intensity profiles along the $x$-axis are to be deconvoluted, this co-ordinate was chosen for continuous dispersion on the photographic emulsion; while $z$ axis and time resolution were obtained in a discontinuous fashion at rather broad intervals using the time-gating optical system shown in Fig. 2. This system is conceptually similar to ones described by SACKS and WALTERs [12] and studied in detail by KLUEPPEL et al.[13].

The apparatus consists of two image transfer optical systems and a triggering optical system. Spherical mirrors $M_{1}$ and $M_{2}(6.3 \mathrm{~cm}$ dia., $61.0 \mathrm{~cm}$ focal length) in a side-by-side configuration image the plasma $P$ onto the vertical plane containing horizontal slit $S_{1}$. This isolates radiation from a thin circular slice of the plasma at some distance $Z_{0}$ from the substrate surface. The thickness of the slice is determined by the width of $S_{1}$ and the magnification of the $M_{1} / M_{2}$ image transfer system. Spherical mirrors $M_{3}$ and $M_{4}(7.0 \mathrm{~cm}$ dia., $44.5 \mathrm{~cm}$ focal length) in an over-and-under configuration image $S_{1}$ onto the vertical plane containing spectrograph slit $S_{2}$ after a $90^{\circ}$ rotation by image rotator $R$. The rotator was constructed from three front-surface plane mirrors [13]. Mirtor $M_{4}$ is rotated about a horizontal axis normal to the optical axis by a $60 \mathrm{~Hz}$ synchronous motor. Thus, the rotated image of $S_{1}$ sweeps across $S_{2}$, and only for the brief interval when the image of $S_{1}$ crosses $S_{2}$ does radiation from the plasma enter the spectrograph.

High spacial resolution along the $z$ co-ordinate is obtained by placing $S_{1}$ at the sagittal image plane of the $M_{1} / M_{2}$ side-by-side mirror system. High time resolution is obtained by focusing the tangential image of $S_{1}$ onto the vertical plane of $S_{2}$ using the $M_{3} / M_{4}$ over-and-under configuration. This tangential image after a $90^{\circ}$ rotation is vertical and thus sweeps across the spectrograph slit as $M_{4}$ is rotated about a horizontal axis. High spacial resolution along the $x$ axis of the plasma, which is needed for reliable deconvolution of the measured intensity profiles, is obtained by bringing to focus the tangential image of the $M_{1} / M_{2}$ system on the photographic emulsion using the combined astigmatism of the $M_{3} / M_{4}$ configuration and the side-by-side mirror configuration of the $1.0 \mathrm{~m}$ Czerny-Turner spectrograph (Jarrell-Ash Model 78-462).

The discharge was initiated by the trigger pulse produced when the image of the incandescent lamp filament ( $F$ in Fig. 1) swept across the photodiode D (EG and G SGD 040A). The $120 \mathrm{~V}, 50 \mathrm{~W}$ projection lamp was focused onto the photodiode through the optical path $L_{1}, M_{4}$ and $L_{2}$. The locations of lenses $L_{1}$ and $L_{2}$ were chosen somewhat arbitrarily to provide a voltage pulse of sufficient amplitude to trigger a

[12] R. D. SACKs and J. P. WALters, Anal. Chem. 42, 61 (1969).

[13] R. J. Klueppel, D. M. Coleman, W. S. Eaton, S. A. Goldstein, R. D. Sacks and J. P. Walters, Spectrochim. Acta 33B, 1 (1978). 
Tektronix Type 547 oscilloscope operated in a single sweep mode. A delayed trigger output pulse from the oscilloscope was used to fire the discharge circuit. By changing the time delay, the time gate position could be set at any point relative to the discharge current wavetrain. Time-gate position reproducibility was better than the $0.5 \mu$ s sampling interval of the Nicolet Model 2090 digital storage oscilloscope used to monitor the discharge current and the time gate position. These waveforms were recorded for every experiment by displaying simultaneously on the oscilloscope the output from a Pearson Model 1025 wide band current transformer and the output from a 1P28 photomultiplier tube located inside the spectrograph so as to respond to stray radiation. Because of the very high radiation intensity, an adequate signal was obtained even when the time gate occurred during a period of very low discharge current. The photomultiplier tube was operated with a $800 \mathrm{~V}$ bias and a $470 \Omega$ load.

\subsection{Optical focusing and calibration}

A grid consisting of $\sim 100 \mu \mathrm{m}$ dia. wires spaced $\sim 300 \mu \mathrm{m}$ between their centers and back illuminated by a $\mathrm{Hg}$ pen lamp was used for focusing individual components as well as the entire system. When the grid was located in the plane of the discharge axis with the wires oriented parallel to the axis and photographed through the entire optical system including the spectrograph, the wire images and spaces between them were completely resolved. Resolution was satisfactory over the entire $20 \mathrm{~mm}$ length of the $\mathrm{Hg}$ emission lines for the $13 \mathrm{~mm}$ wide region of the focal plane used to record spectra for deconvolution.

The $z$ axis spacial resolution of the discharge plasma was limited by the $200 \mu \mathrm{m}$ width of slit $S_{1}$. With smaller slit widths, insufficient exposure was obtained on the Kodak Royal-X Pan emulsion. For most experiments, the $M_{1} / M_{2}$ mirror system was used with unit magnification. This gave a $z$ axis resolution of $0.2 \mathrm{~mm}$. For some measurements, a 0.33 magnification was employed resulting in a $z$ axis resolution of $0.6 \mathrm{~mm}$. Since the exploding thin film plasma is physically quite large, measurements in this survey study were made at $1.0 \mathrm{~mm}$ intervals along the $z$ axis, and thus even $0.6 \mathrm{~mm}$ resolution is satisfactory.

The spectrograph slit was $100 \mu \mathrm{m}$ wide, and the $M_{3} / M_{4}$ system magnification was unity for all experiments. This results in a calculated time gate width of $0.88 \mu \mathrm{s}$. The measured time gate width ranged from 0.95 to $1.20 \mu \mathrm{s}$. This is shown in relation to the discharge current waveform in Fig. 3. Clearly, this time resolution is adequate for the relatively slowly varying discharge current. The spectrograph was used in the second order with a reciprocal linear dispersion of $0.8 \mathrm{~nm} / \mathrm{mm}$.

It should be noted that focusing and alignment tolerances are quite stringent, since an error of only $0.2 \mathrm{~mm}$ in the distance from $M_{4}$ to $S_{2}$ results in a two-fold increase in effective time-gate width. In addition a $0.86^{\circ}$ rotational misalignment of $S_{2}$ and the image of $S_{1}$ results in about a $1 \mu$ s variation in time-gate location along each spectral

(a)

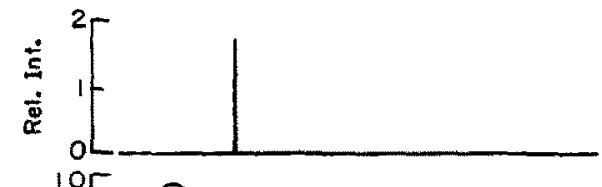

(b)

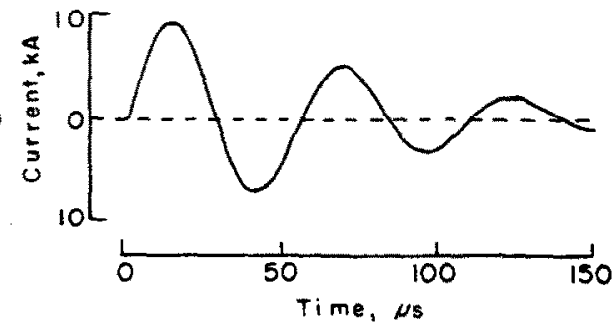

Fig. 3. Oscilloscope traces from (a) the output of the photomultiplier tube inside the spectrograph used to monitor the time gate width and location, and (b) the output of the current transformer used to monitor discharge current. 
line [14]. Shot-to-shot time-gate location reproducibility and predictability were relatively unimportant for these non-repetitive discharges since a time-gate monitor pulse and a current waveform were obtained for every experiment.

\subsection{Data acquisition procedures}

Photographic processing is discussed elsewhere [15]. Emulsion calibration was accomplished with a computer program routinely used in this laboratory [16]. Timegated, spacially resolved photographic spectra were obtained for various heights above the substrate surface. Spectra from four explosions were obtained at each temporal and axial location, and the one showing the greatest symmetry with respect to the discharge axis was used for deconvolution. Microdensitometer traces were obtained across the profiles of selected lines at 0.5 or $1.0 \mathrm{~mm}$ intervals along the line lengths. A Joyce-Loebl Mark IIIB recording microdensitometer using a $20 \mu \mathrm{m} \times 20 \mu \mathrm{m}$ scanning aperture was used for all measurements.

All microdensitometer traces were digitized with a Model HW-2-48 digitizer (Summagraphics Corp.). The spacial resolution of the digitizer was set to correspond to $20 \mu \mathrm{m}$ per point on the emulsion. Seven points were recorded for each line profile with the first and the last points in the region of continuum background well removed from the line of interest. Data were stored on a floppy disk and transferred to the Amdahl $470 \mathrm{~V} / 8$ main computer facility by a PDP-11 minicomputer. Since a very large amount of data was involved in these studies, a computerized editing routine was developed to check for missing and redundant data.

Since the emulsion response is non-linear, each of the seven optical density values across a spectral line profile was converted individually to an intensity value. Background intensity was obtained from a linear interpolation of the end points. Background corrected intensity values were obtained by equation (16):

$$
I\left(\lambda_{j}\right)=\psi\left(D_{i}\right)-\left[\frac{\psi\left(D_{7}\right)-\psi\left(D_{1}\right)}{\lambda_{7}-\lambda_{1}}\right]\left(\lambda_{j}-\lambda_{1}\right)-\psi\left(D_{1}\right),
$$

where $\psi$ is the transformation function used to convert optical density $D_{j}$ into relative intensity, and $\lambda_{i}$ is the wavelength co-ordinate of the measurement. RomberG integration [17] of the seven intensity values for each line profile then was used to obtain the total intensity integrated across the line profile.

The plot of these integrated intensity values as a function of position along the length of a spectral line results in the measured intensity distribution, an example of which is shown in Fig. 4a. These intensity distributions were symmetrized by averaging the mirror images about the $x=0$ axis. This is shown in Fig. $4 \mathrm{~b}$.

\subsection{Deconvolution procedures}

Deconvolution of the symmetrized intensity distributions was accomplished using the inverted form of the Abel integral equation [18]

$$
J(\bar{r})=-\frac{1}{\pi} \int_{r}^{x} \frac{(\mathrm{d} I(x) / \mathrm{d} x)}{\sqrt{ }\left(x^{2}-r^{2}\right)} \mathrm{d} x,
$$

where $I(x)$ is the measured intensity distribution. A number of computer techniques for obtaining $J(r)$ based on numerical integration[19,20] and geometric algorithms $[12,21]$ have been described. Numerical integration was used in this study.

[14] S. Y. Suh, Ph.D. Dissertation. University of Michigan, Ann Aroor. MI (1980).

[15] R. D. SACKS and C. S. LinG, Appl. Spectrosc. 33. 258 (1979).

[16] J. A. Holcombe, D. W. Brinkman and R. D. SaCks, Anal. Chem. 47, 441 (1975).

[17] C. E. FröberG, Introduction to Numerical Analysis. Addison-Wesley. Reading. MA (1965).

[18] H. Margenau and G. M. Murphy, The Mathematics of Physics and Chemistry. Van Nostrand, Princeton (1956).

[19] W. L. BARR, J. Opt. Soc. Am. 52, 885 (1962).

[20] C. J. Cremers and R. C. Birkebak, Appl. Optics 5, 1057 (1966).

[21] A. Scheeline and J. P. Walters, Anal. Chim. Acta 95. 59 (1977). 
(a)

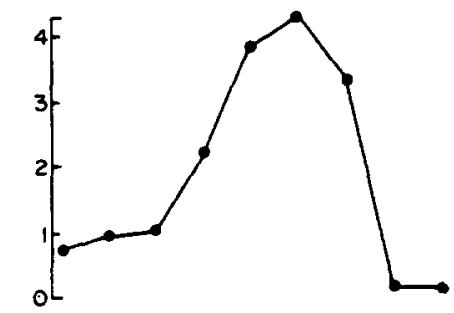

(b)

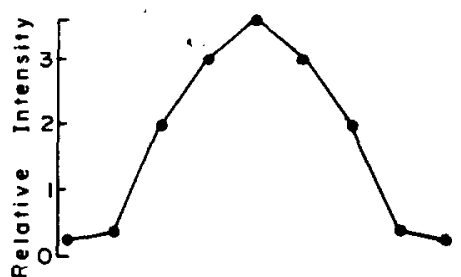

(c)

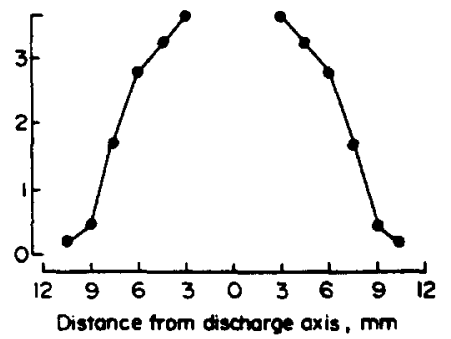

Fig. 4. (a) Measured intensity distribution along the length of a temporally and spacially resolved $\mathrm{Fe}$ (II) line. Each point represents the relative intensity integrated across the line profile; (b) intensity distribution from (a) symmetrized by averaging the mirror images about the $x=0$ axis;

(c) deconvoluted intensity profile showing radial dependence of spectral emissivity.

Equation (17) has a singularity at the lower integration limit. While this singularity is integrable, excessive computer time is required. The integrand singularity can be removed except at the origin using integration by parts to convert equation (17) into equation (18):

$$
J(r)=\frac{1}{\pi} \int_{r}^{\infty} V\left(x^{2}-r^{2}\right)\left[\frac{x\left(\mathrm{~d}^{2} I(x) / \mathrm{d} x^{2}\right)-(\mathrm{d} I(x) / \mathrm{d} x)}{x^{2}}\right] \mathrm{d} x
$$

When test data sets were integrated using equations (17) and (18), identical values of $J(r)$ were obtained, but the required computer time was significantly smaller with equation (18).

The symmetrized intensity profiles were smoothed further using the cubic spline method [22]. This method was chosen since the smoothed curve is continuously differentiable through the second derivative. This is necessary for equation (18).

While it is straightforward to obtain exact values of the derivatives from the smoothed intensity profiles, artifacts may be introduced. Therefore, a sort of average derivative over the interval $\Delta x$ was obtained according to equation (19):

$$
\frac{\mathrm{d} I(x)}{\mathrm{d} x}=\frac{I(x+\Delta x)-I(x-\Delta x)}{2 \Delta x} .
$$

The derivative obtained in this way is not critically dependent on the interpolation technique. While this local average derivative may be useful in reducing random noise in the data and artifacts in the smoothing procedure, spacial resolution is sacrificed. With $\Delta x=0.25 \mathrm{~mm}$ and an optical system magnification of 0.33 , spacial resolution in the plasma is about $0.75 \mathrm{~mm}$.

[22] L. F. Shampine and R. C. Allen, Jr., Numerical Computing. W. B. Saunders. Philadelphia (1973). 
Once the derivatives were obtained, equation (17) or (18) was integrated using Gauss-Kronrod rules [23,24] and a queue algorithm [25]. This integration routine detects and processes endpoint singularities and round-off errors in the integrand values. The emissivity at the origin was not evaluated because of error accumulation. The zone near the radiating boundary also was discarded since this region is too sensitive to the curve smoothing procedure. An example of a deconvoluted intensity profile is shown in Fig. 4c. Similar profiles were obtained for the nine $\mathrm{Fe}(\mathrm{II})$ and two $\mathrm{Fe}(\mathrm{I})$ lines listed in Table 1.

The relative emissivity data as $\ln \left(\lambda J^{\prime} / g A^{\prime}\right)$ for the $\mathrm{Fe}(\mathrm{II})$ lines were plotted as functions of the upper state excitation energy for the nine transitions. An example is shown in Fig. 5. From the slope of the curve (linear least squares fit), the plasma temperature was obtained. The two $\mathrm{Fe}(\mathrm{I})$ lines in Table 1 were used to evaluate degree of ionization through equation (3). The partition functions for $\mathrm{Fe}$ and $A^{\prime}$ values were obtained from Refs [26] and [27], respectively.

Table 1. Iron lines in the $315-326 \mathrm{~nm}$ region

\begin{tabular}{|c|c|c|c|c|c|c|}
\hline \multirow{2}{*}{$\begin{array}{l}\text { Emitting } \\
\text { species }\end{array}$} & \multirow{2}{*}{$\begin{array}{l}\text { Wavelength } \\
\text { (nm) }\end{array}$} & \multirow{2}{*}{$\begin{array}{l}\text { Transition } \\
\text { Probability } \\
\left(\mathbf{s}^{-1}\right)\end{array}$} & \multicolumn{2}{|c|}{ Lower state } & \multicolumn{2}{|c|}{ Upper State } \\
\hline & & & Energy (eV) & J-value & Energy (eV) & J-value \\
\hline$F e(I I)$ & 325.91 & $1.9 \times 10^{8}$ & 3.90 & 3.5 & 7.71 & 4.5 \\
\hline $\mathrm{Fe}(\mathrm{I})$ & 324.70 & $7.4 \times 10^{6}$ & 2.20 & 2.0 & 6.01 & 1.0 \\
\hline Fe(II) & 324.37 & $1.5 \times 10^{8}$ & 4.15 & 4.5 & 8.00 & 3.5 \\
\hline$F e(I I)$ & 323.78 & $3.6 \times 10^{8}$ & 3.89 & 0.5 & 7.72 & 1.5 \\
\hline Fe(II) & 322.77 & $1.6 \times 10^{8}$ & 1.67 & 2.5 & 5.51 & 3.5 \\
\hline $\operatorname{Fe}(I I)$ & 321.33 & $1.3 \times 10^{8}$ & 1.69 & 1.5 & 5.55 & 2.5 \\
\hline$F \in(I)$ & 319.32 & $4.5 \times 10^{7}$ & 0 & 4.0 & 3.88 & 4.0 \\
\hline$F e(I I)$ & 318.67 & $1.1 \times 10^{8}$ & 1.69 & 1.5 & 5.58 & 1.5 \\
\hline$F e(I I)$ & 317.75 & $2.0 \times 10^{8}$ & 3.90 & 3.5 & 7.80 & 3.5 \\
\hline$F e(I I)$ & 316.79 & $3.2 \times 10^{8}$ & 3.81 & 3.5 & 7.73 & 3.5 \\
\hline$F e(I I)$ & 315.42 & $3.9 \times 10^{8}$ & 3.77 & 4.5 & 7.70 & 4.5 \\
\hline
\end{tabular}

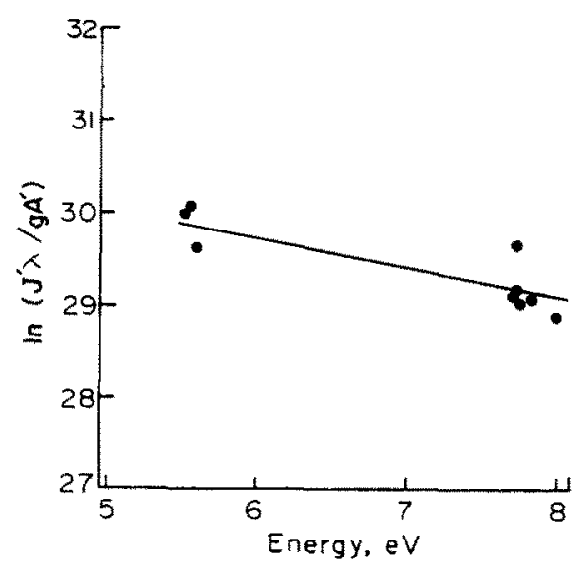

Fig. 5. Boltzmann graphical method plot for the nine Fe(II) lines. Effective electronic excitation temperature is estimated from the linear least squares slope.

[23] J. Todo, Survey of Numerical Analysis. McGraw-Hill, New York (1962).

[24] D. K. KahaneR, Comparison of Numerical Quadrature Formulas. Los Alamos Scientific Laboratory Report LA-4137.

[25] QKSQ, University of Michigan Computing Center Library Routine.

[26] H. W. Drawin and P. Felenbok, Data for Plasmas in Local Thermal Equilibrium. Gauthier-Villars, Paris (1965).

[27] R. L. Kurucz and E. Peytremann, Smithsonian Astrophysical Observatory Special Report No. 362 (1975). 


\subsection{Experimental conditions and procedures}

Apparatus and procedures for the production of thin $\mathrm{Ag}$ films on polycarbonate membrane filters have been described in detail $[1,28]$. Table 2 summarizes properties of the thin films and substrates along with the discharge conditions. Iron samples were prepared from high-purity metallic powder suspended in isopropyl alcohol. Only Fe particles which passed through a $5 \mu \mathrm{m}$ pore dia. sieve were used to ensure complete sample atomization [1]. An aliquot of the stirred suspension containing $5 \mu \mathrm{g}$ of Fe was deposited near the center of each film. The alcohol was evaporated under a heat lamp.

\section{Operation of the Coaxial System}

The coaxial electrode geometry used in these studies results in a radially directed current path through the thin film and, after vaporization of the film, through the plasma. Thus the current density decreases with increasing distance from the center electrode. This has a profound effect on the vaporization process. For the linear film geometry used with previous studies [1], current density is nearly the same over the entire film. This results in the rapid conversion of the entire film from a conducting solid or liquid to a gaseous dielectric. This results in a sudden decrease in discharge current and the simultaneous development of a large voltage transient. This voltage transient often results in the dielectric breakdown of the gas surrounding the expanding metal vapor cloud [29]. The result is the appearance of neutral atom and ion lines from the surrounding gas, an increase in continuum background intensity and a significant decrease in shot-to-shot reproducibility [2].

With the coaxial geometry, the radial variation in current density results in earlier vaporization of the film in the vicinity of the center electrode. An arc may develop from the center electrode to the rapidly moving edge of the unvaporized film. When the vaporization front reaches the outer electrode, full plasma conduction begins. This gradual transfer of current conduction from the thin film to the vapor cloud prevents the development of a voltage transient and the attendant breakdown of the surrounding gas. The resulting increase in shot-to-shot reproducibility was indeed welcome for the studies reported here.

Table 2. Summary of thin film/substrate properties and discharge conditions

\begin{tabular}{|c|c|}
\hline \multicolumn{2}{|l|}{ Thin Ag films } \\
\hline Thickness*,$\AA$ & 280 \\
\hline Mass vaporized, ${ }^{\dagger} \mathrm{Hg}$ & 80 \\
\hline Diameter, mm & 19 \\
\hline \multicolumn{2}{|c|}{ Polycarbonate membrane filter substrates } \\
\hline Thickness, mm & 0.01 \\
\hline Diameter, mm & 47 \\
\hline Pore diameter, um & 0.4 \\
\hline Pore density, $\mathrm{cm}^{-2}$ & $10^{8}$ \\
\hline \multicolumn{2}{|l|}{ Discharge conditions } \\
\hline Plasma support gas & $608 \mathrm{Ar}-408 \mathrm{O}_{2}$ \\
\hline Ambient pressure, torr & 700 \\
\hline Charging vol tage, $\mathrm{kV}$ & 4.5 \\
\hline Capacitance, i $\mathbf{F}$ & 22.5 \\
\hline Energy, I & 228 \\
\hline Ringing frequency, $\mathrm{kHz}$ & 19 \\
\hline
\end{tabular}

[28] E. M. ClaRK, Ph.D. Dissertation. University of Michigan, Ann Arbor, MI (1979).

[29] R. D. SACKS and J. A. Holcombe, Appl. Spectrosc. 28, 518 (1974). 
Figure 6 shows the discharge current waveform along with radiation intensity profiles from the coaxial film system. Figure 6a shows the continuum background near $388.9 \mathrm{~nm}$. The background intensity is quite reproducible with relative standard deviations of about $\pm 2 \%$. Figure $6 \mathrm{~b}$ shows the background corrected $\mathrm{Mn} 388.9 \mathrm{~nm}$ line from four consecutive shots each using $1.0 \mu \mathrm{g} \mathrm{Mn}$. In general, the relative standard deviations of the intensities were around $\pm 5 \%$. The $\mathrm{Mn} 388.9 \mathrm{~nm}$ line is really a complex group of unresolved lines representing neutral atom, singly ionized, and doubly ionized Mn species. For the intensity profiles in Fig. 6, no spacial resolution was employed, and the spectrometer viewed the entire plasma volume. The intensities of both the analysis line and the continuum background oscillate significantly with the discharge current. However, line-to-background ratios tend to be largest during intervals of low discharge current. Similar results were observed for a linear plasma geometry [2].

\section{Thermodynamic Properties}

When spectroscopic methods are applied to the determination of thermodynamic plasma properties, several points should be considered. First, LTE may not be achieved. Thus, the temperature may not be rigorously defined. The best hope would be that LTE is nearly achieved over the range of excited states used for temperature measurements. The temperatures reported here then have a very restrictive definition.

Second, self-absorption almost always occurs. While correction methods for selfabsorption have been applied successfully $[30,31]$, experimental difficulties would be considerable for the transient, nonrepetitive discharges described here. SCHEELINE and WALTERS [32] used computer modeling techniques to demonstrate significant emis-

(a)
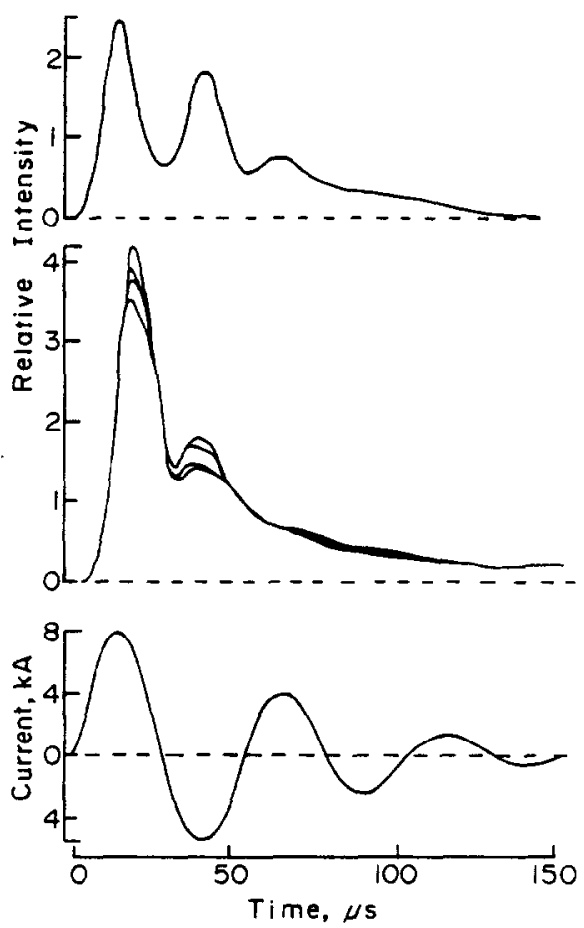

Fig. 6. Discharge current and radiation waveforms form: (a) continuum background; and (b) the $\mathrm{Mn} 388.9 \mathrm{~nm}$ line from four consecutive $1.0 \mu \mathrm{g}$ Mn samples.

[30] M. P. Freeman, J. Opt. Soc. Am. 50,826 (1960).

[31] M. P. Freeman and S. Katz, J. Opt. Soc. Am. 53, 1172 (1963).

[32] A. Scheeline and J. P. Walters, Anal. Chem. 48, 1519 (1976). 
sivity profile distortions when self-absorption occurs but is not accounted for in the deconvolution procedure. To minimize problems from self-absorption, no resonance transitions were used for temperature measurements. For the nine Fe(II) lines listed in Table 1 , the lowest energy lower state is $1.7 \mathrm{eV}$ above the ion ground state. While no detailed line profile studies were attempted, visual examination of microdensitometer traces across selected $\mathrm{Fe}(\mathrm{II})$ lines revealed no obvious self absorption.

The lack of complete plasma symmetry also should be noted since it results in emissivity profile errors during the deconvolution process [32]. While this problem has been treated with some success by simultaneously recording spacially resolved spectra along two orthogonal axes through the plasma[31], the instrumentation requirements would be excessive for the studies reported here.

The final point to note is that radiation dispersion along temporal and spacial dimensions results in poor reproducibility and relatively low intensities. The interpretation of the data presented in this section should be limited by these uncertainties.

\subsection{Temperature}

The nine Fe(II) lines which were found to have suitable intensities for temperature calculations and for which published transition probability values were considered reliable all belong to two groups of multiplets separated by about $2.2 \mathrm{eV}$ (see Fig. 5). While this might result in somewhat more reliable determination of the Boltzmann graph slope, it is unfortunate in that little can be ascertained regarding the degree of LTE in the plasma.

Table 3 shows the computed electronic temperature values for various heights above the electrode surface and for various times during the first current halfcycle. These electronic excitation temperatures generally range from about $7000 \mathrm{~K}$ to $15000 \mathrm{~K}$ with the higher values generally occurring near the time of peak discharge current $(-13 \mu \mathrm{s})$. These values are in the same range as the gas kinetic temperatures estimated by HoLCOMвE [33] for the plasmas generated by electrically exploded Ag wires. No other temperature studies of electrically vaporized thin film plasmas could be found in the literature.

While the values in Table 3 are not sufficient for a characterization of the horizontal temperature gradient, the few values obtained $6 \mathrm{~mm}$ from the discharge axis are quite similar to the $3 \mathrm{~mm}$ values. A few experiments with the center electrode initially cathodic resulted in significantly higher temperatures than the corresponding values in Table 3 (center initially anodic), especially near the time of the current peak. Because of the radial current conduction path in the plasma, greater current density is expected near the center electrode. When this electrode is negative (cathodic) sample vapor ions may be drawn into regions of greater current density. For all of the

Table 3. Electronic excitation temperature in exploding thin film plasmas

\begin{tabular}{|c|c|c|c|c|c|c|}
\hline Current (kA) & 7.9 & 9.2 & 8.7 & 7.4 & 5.2 & 0.12 \\
\hline Time ( $\mu s$ ) & 8.5 & 13.0 & 15.5 & 18.0 & 21.0 & 25.5 \\
\hline Height $(\mathrm{mm})$ & \multicolumn{6}{|c|}{ Temperature $(\mathrm{K}) \times 10^{-3}$ * } \\
\hline 2.0 & 14 & 15 & & & & \\
\hline 3.0 & & $\begin{array}{l}12 \\
(10)\end{array}$ & 9.1 & & 12 & \\
\hline 6.0 & & 11 & & 8.2 & $(7.3)$ & $\begin{array}{r}9.0 \\
(8.9)\end{array}$ \\
\hline 10.0 & & & & & 9.2 & 6.7 \\
\hline
\end{tabular}

\footnotetext{
*Parenthetical values obtained $6 \mathrm{~mm}$ from discharge axis; all other values obtained $3 \mathrm{~mm}$ from axis.
}

[33] J. A. Holcombe. Ph.D. Dissertation, University of Michigan, Ann Arbor, MI (1974). 
temperatures reported here, it should be recalled that vertical distance is measured relative to the upper surface of the outer electrode. Since this is about $3 \mathrm{~mm}$ above the plane of the film and the center electrode higher temperatures than those reported in Table 3 may occur in the very high current density region near the center electrode.

The uncertainty in temperature measurements was estimated from equations (7) and (10) using \pm 0.5 for $\epsilon_{A}, \pm 0.3$ for $\epsilon_{J}$ and $2.2 \mathrm{eV}$ for $\Delta E$. The resulting values for $\epsilon_{T}$ range from $\pm 24 \%$ for a temperature of $10,000 \mathrm{~K}$ to $\pm 35 \%$ for a temperature of $15,000 \mathrm{~K}$. Both $\epsilon_{A}$ and $\epsilon_{J}$ are difficult to estimate, and values were chosen somewhat arbitrarily. Absolute transition probability values in the literature are notoriously inaccurate and inconsistent [34]. GolDBERG et al.[35] give an excellent account of the transition probability problem in obtaining reliable temperature measurements. Since the nine Fe(II) transition probability values used in these studies were obtained on the same scale, and since only relative values are used for the Boltzmann graph, the value \pm 0.5 for $\epsilon_{A}$ may be quite conservative. For all temperature values reported in Table 3, the temperature uncertainty obtained from the standard deviation of the Boltzmann graph regression estimate is within the range predicted by equations (7) and (10).

\subsection{Degree of ionization}

From the electronic excitation temperatures in Table 3, the degree of ionization was evaluated from the ratio of neutral atom to ion line intensities. The two $\mathrm{Fe}$ (I) lines (see Table 1) were chosen to have upper level excitation energy comparable to the $\mathrm{Fe}$ (II) lines used for temperature measurements. For each temperature entry in Table 3,18 degrees of ionization values were computed using all possible neutral atom/ion intensity ratios from the lines in Table 1 . Any values within a group of 18 which deviated from the mean by more than three standard deviations were rejected and a new mean computed. These final values are presented in Table 4 for various distances above the electrode surface and for various times during the first current halfcycle. It is interesting to note that when the degree of ionization values obtained from the two neutral-atom lines are compared, there is no statistically significant difference in mean values, but the relative standard deviations generally are larger with the resonance neutral-atom line $(319.32 \mathrm{~nm})$. This suggests that self-absorption may not be sufficient to cause major errors in the studies reported here.

The degree of ionization increases significantly with increasing distance from the electrode surface despite decreasing temperature. This suggests that ionization is controlled more by the size and location of the current conduction zone and its associated high electron density than by the plasma temperature. Further, the degree of ionization is lower near the time of peak current $(\sim 13 \mu \mathrm{s})$ in all observation zones,

Table 4. Extent of ionization in exploding thin film plasmas

\begin{tabular}{|c|c|c|c|c|c|c|}
\hline Current (kA) & 7.9 & 9.2 & 8.7 & 7.4 & 5.2 & 0.12 \\
\hline Time $(\mu s)$ & 8.5 & 13.0 & 15.5 & 18.0 & 21.0 & 25.5 \\
\hline Height (mm) & & & Fraction & ionized* & & \\
\hline 1.0 & 0.63 & 0.68 & & & & \\
\hline 3.0 & & $\begin{array}{c}0.71 \\
(0.89)\end{array}$ & 0.91 & & 0.78 & \\
\hline 6.0 & & & & 0.95 & $\begin{array}{c}0.98 \\
(0.97)\end{array}$ & $\begin{array}{c}0.94 \\
(0.97)\end{array}$ \\
\hline 10.0 & & & & & 0.93 & 0.99 \\
\hline
\end{tabular}

*Parenthetical values obtained $6 \mathrm{~mm}$ from discharge axis; all other values obtained $3 \mathrm{~mm}$ from axis.

[34] H. Chuang, Appl. Optics 4, 1589 (1965).

[35] L. Goldberg, E. A. Müller and L. H. Aller, Astrophys. Suppl. 45, 1 (1960). 
again despite higher temperature at the time of peak current. This also can be explained by the mass action effect of electron density on ionization equilibrium. The greater degree of ionization observed $6 \mathrm{~mm}$ from the discharge axis relative to $3 \mathrm{~mm}$ at the $3 \mathrm{~mm}$ axial location is probably the result of the radial variation of current density expected with the coaxial electrode geometry.

A general increase in degree of ionization with increasing time also is observed in Table 4 independent of observation zone and discharge current. This may result from volume dilution of analyte species by diffusive processes with a corresponding decrease in charge density.

From equations (13) and (14) and using the same values of $\epsilon_{j}$ and $\epsilon_{A}$ as used for evaluating $\epsilon_{T}$, values of $\epsilon_{\alpha}$ range from $\pm 6 \%$ for an $\alpha$ of 0.90 to $\pm 58 \%$ for an $\alpha$ of 0.50 . Since most of the data indicate a high degree of ionization, the values of $\alpha$ reported here should be quite reliable. Again, the relative standard deviations of $\alpha$ values obtained from the various neutral-atom/ion line pairs were within the corresponding $\epsilon_{a}$ range.

Since the degree of ionization values in Table 4 are all greater than 0.5 , it is not surprising that ion lines typically have resulted in greater powers of detection and greater analytical precision than neutral-atom lines [1]. Since the degree of ionization of $\mathrm{Fe}$ in Table 4 is greater than 0.9 for discharge currents as low as $120 \mathrm{~A}$, it is unlikely that time-gated integration[2] would significantly increase the utility of neutral atom lines.

\subsection{Electron number density}

Electron number density values obtained from the temperature and degree of ionization values in Tables 3 and 4, respectively, are presented in Table 5. It should be emphasized that the degree of ionization of Fe presented in Table 4 does not necessarily refiect the charge density in the plasma since the Fe ions probably are not the major charge carriers. The degree of ionization simply depends on the temperature and the electron number density through the Saha equilibrium. The electron density values in Table 5 relate more directly to current density in the discharge.

As expected, the electron density is greatest near the electrode surface and at the time of peak discharge current. The electron density also is greater $3 \mathrm{~mm}$ from the discharge axis than $6 \mathrm{~mm}$ from the axis. This is probably the result of the coaxial electrode geometry. It is interesting to note that the electron pressures calculated from the electron number densities are much greater than ambient gas pressure at peak current and near the electrode surface. Using the ideal gas law, the electron pressure at $1 \mathrm{~mm}$ and $13 \mu \mathrm{s}$ is calculated to be about 10 atm. This indicates that there exists a considerable magnetic pressure in order to maintain such a high electron

Table 5. Electron concentrations in exploding thin film plasmas

\begin{tabular}{|c|c|c|c|c|c|c|}
\hline Curzent $(\mathrm{kA})$ & 7.9 & 9.2 & 8.7 & 7.4 & 5.2 & 0.12 \\
\hline Time (us) & & 13.0 & 15.5 & 18.0 & 21.0 & 25.5 \\
\hline Height (mm) & & \multicolumn{5}{|c|}{ Electron Concentration $\left(\mathrm{cm}^{-3}\right) *$} \\
\hline 1.0 & $4 \times 10^{18}$ & $5 \times 10^{18}$ & & & & \\
\hline 3.0 & & $\begin{array}{l}1 \times 10^{18} \\
\left(9 \times 10^{16}\right)\end{array}$ & $2 \times 10^{16}$ & & $8 \times 10^{27}$ & \\
\hline 6.0 & & $2 \times 20^{17}$ & & $4 \times 10^{2}$ & $\begin{array}{c}4 \times 10^{25} \\
\left(1 \times 10^{15}\right.\end{array}$ & $\begin{array}{l}1 \times 10^{16} \\
\left(6 \times 10^{15}\right)\end{array}$ \\
\hline 10.0 & & & & & $2 \times 10^{26}$ & $6 \times 10^{13}$ \\
\hline
\end{tabular}

*Parenthetical values obtained $6 \mathrm{~mm}$ from discharge axis; all other values obtained $3 \mathrm{~mm}$ from axis. 
pressure. However, near the end of the first current halfcycle, the electron pressure drops to $\sim 10^{-3} \mathrm{~atm}$.

The values of electron density probably are reliable only within an order of magnitude. From equation (15), the uncertainty associated with the electron density measurement is significantly greater than that of the ion/neutral atom density ratio from which the former is derived. This may explain in part why literature values of electron density are widely scattered.

\section{Conclusions}

The conditions under which LTE is to be expected in a plasma have been investigated theoretically by BATES et al. $[36,37]$. Assuming that collisional excitation across the largest energy gap in the energy level scheme is the weakest link in the chain of energy redistribution processes leading to LTE, A necessary condition for LTE in a collision-dominated plasma was derived by GRIEM [38] and WILSON [39] as

$$
n_{e} \geq 10^{12} T^{1 / 2}(\Delta E)^{3},
$$

where $n_{e}$ is the electron density, and $\Delta E$ is the largest energy gap. Even for an energy gap as large as $10 \mathrm{eV}$ and a plasma temperature of $10,000 \mathrm{~K}$, the required electron density $\left(10^{17} \mathrm{~cm}^{-3}\right)$ may be present in the exploding thin film plasma. Clearly, however, much more experimental data are required for a definitive evaluation.

The temperature and electron density values reported in Tables 3 and 5 , respectively, are similar to values reported for the spark discharge. As early as 1945 CRAGGS and MEEK [40] reported electronic temperature values of $10,000-15,000 \mathrm{~K}$ at peak current and $8000-10,000$ at zero current for a high-voltage spark discharge. The corresponding electron density values were reported as $10^{17}$ and $10^{15} \mathrm{~cm}^{-3}$, respectively. While these values may be suspect, they do suggest similarities in the excitation environment in the current channel regions of the exploding thin film and high-voltage spark discharges.

Acknowledgement-The authors acknowledge support of this study by the National Science Foundation through grant No. CHE 78-25542.

[36] D. R. Bates, A. E. Kingston and R. W. P. McWhirter, Proc. Roy. Soc. 267A, 297 (1962).

[37] D. R. Bates, A. E. Kingston and R. W. McWhiRTer, Proc. Roy. Soc. 270A, 155 (1962).

[38] H. R. Griem, Phys. Rev. 131, 1170 (1963).

[39] R. WILSON, J. Quant. Spectr. Radiat. Transfer 2, 447 (1962).

[40] J. D. Craggs and J. M. Meek, Proc. Roy. Soc. 186A, 241 (1945). 